Document downloaded from:

http://hdl.handle.net/10251/83059

This paper must be cited as:

Ballester-Bolinches, A.; Esteban Romero, R.; Qiao, S. (2016). A note on a result of Guo and Isaacs about p-supersolubility of finite groups. Archiv der Mathematik. 106(6):501-506. doi:10.1007/s00013-016-0901-7.

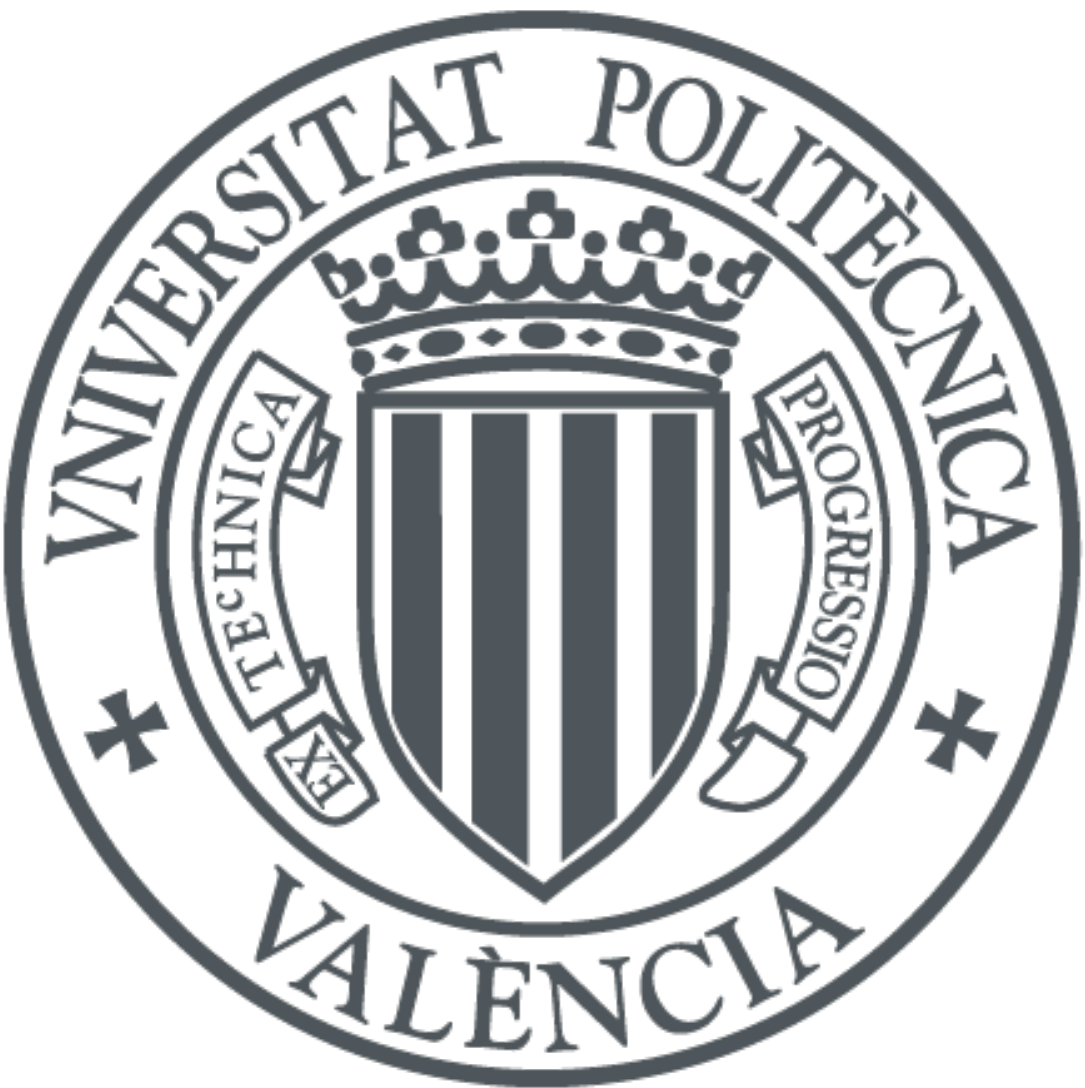

The final publication is available at

https://link.springer.com/article/10.1007/s00013-016-0901-7

Copyright Springer Verlag (Germany)

Additional Information

The final publication is available at Springer via http://dx.doi.org/10.1007/s00013-016-09017 


\title{
A note on a result of Guo and Isaacs about $p$-supersolubility of finite groups
}

\author{
Adolfo Ballester-Bolinches* Ramón Esteban-Romero ${ }^{\dagger}$ \\ ShouHong Qiao ${ }^{\ddagger}$
}

\begin{abstract}
In this note, global information about a finite group is obtained by assuming that certain subgroups of some given order are S-semipermutable. Recall that a subgroup $H$ of a finite group $G$ is said to be S-semipermutable if $H$ permutes with all Sylow subgroups of $G$ of order coprime to $|H|$. We prove that for a fixed prime $p$, a given Sylow $p$-subgroup $P$ of a finite group $G$, and a power $d$ of $p$ dividing $|G|$ such that $1 \leq d<|P|$, if $H \cap \mathrm{O}^{p}(G)$ is S-semipermutable in $\mathrm{O}^{p}(G)$ for all normal subgroups $H$ of $P$ with $|H|=d$, then either $G$ is $p$-supersoluble or else $\left|P \cap \mathrm{O}^{p}(G)\right|>d$. This extends the main result of Guo and Isaacs in Arch. Math. (Basel), 105, 215-222 (2015). We derive some theorems that extend some known results concerning S-semipermutable subgroups.

Mathematics Subject Classification (2010): 20D10, 20D20.

Keywords: finite group, $p$-supersoluble group, S-semipermutable subgroup.
\end{abstract}

\section{Introduction}

All groups mentioned are implicitly assumed to be finite.

*Departament d'Àlgebra, Universitat de València; Dr. Moliner, 50; 46100 Burjassot, València, Spain; email: Adolfo.Ballester@uv.es

$\dagger$ Institut Universitari de Matemàtica Pura i Aplicada, Universitat Politècnica de València; Camí de Vera, s/n; 46022 València, Spain; email: resteban@mat.upv.es. Current address: Departament d'Àlgebra, Universitat de València; Dr. Moliner, 50; 46100 Burjassot, València, Spain; email: Ramon.Esteban@uv.es

${ }^{\ddagger}$ School of Applied Mathematics, Guangdong University of Technology, Guangzhou 510006 People's Republic of China; email: qshqsh513@163.com

$\S$ Corresponding author 
Recall that two subgroups $H$ and $K$ of a group $G$ are said to permute if $H K=K H$, that is, $H K$ is a subgroup of $G$. A subgroup $H$ of a group $G$ is said to be $S$-permutable ([5], see also [1, Section 1.2]) in $G$ if $H$ permutes with all Sylow subgroups of $G$, and is said to be $S$-semipermutable in $G$ ([9]) if $H$ permutes with all Sylow $q$-subgroups of $G$ for the primes $q$ not dividing $|H|$.

Skiba, in his seminal paper [8], introduced the following subgroup embedding property: a subgroup $H$ of a group $G$ is said to be weakly S-permutable in $G$ if there is a subnormal subgroup $T$ of $G$ such that $G=H T$ and $H \cap T \leq H_{s G}$, where $H_{s G}$ is the largest $S$-permutable subgroup of $G$ contained in $H$. This embedding property of subgroups has a strong structural impact and generalises many other known properties.

Recently, Guo and Isaacs considered in [2] the condition $U \cap H \unlhd U$, with $U=\mathrm{O}^{p}(G)$, for a subgroup $H$ of order $d$, where $d>1$ is a power of $p$ such that $d$ divides $|G|$ and $p$ is a prime that we hold fixed. This condition is less restrictive than weak S-permutability [2, Lemma A] and allows them to prove the following result.

Theorem 1 ([2, Theorem B] $)$. Let $P \in \operatorname{Syl}_{p}(G)$ and let $d$ be a power of $p$ such that $1 \leq d<|P|$. Let $U=\mathrm{O}^{p}(G)$, and assume that $H \cap U \unlhd U$ for all subgroups $H \unlhd P$ with $|H|=d$. Then either $G$ is p-supersoluble, or else $|P \cap U|>d$.

An interesting idea of [2] is that in the hypothesis of the theorem, only the normal subgroups of order $d$ are considered, not necessarily the set of all subgroups of order $d$, at the drawback of obtaining as a conclusion either $p$-supersolubility or a restriction on the order of the Sylow $p$-subgroup of $\mathrm{O}^{p}(G)$.

We prove an analogous result, but instead of assuming that all subgroups $H \cap U$ are normal in $U$, we assume that all of them are S-semipermutable in $U$. Our starting point is the following observation. Let $A$ be a $p$-subgroup of a group $G$. By [1, Lemma 1.2.16], $A \cap \mathrm{O}^{p}(G) \unlhd \mathrm{O}^{p}(G)$ if and only if $A \cap \mathrm{O}^{p}(G)$ is S-permutable in $G$. If $A$ is contained in $\mathrm{O}^{p}(G)$, and $q$ is a prime different from $p$, then every Sylow $q$-subgroup of $G$ is contained in $\mathrm{O}^{p}(G)$. Hence $A$ is S-semipermutable in $G$ if and only if $A$ is $\mathrm{S}$-semipermutable in $\mathrm{O}^{p}(G)$.

We prove:

Theorem 2. Let $P \in \operatorname{Syl}_{p}(G)$ and let $d$ be a power of $p$ such that $1 \leq d<|P|$. Assume that $H \cap \mathrm{O}^{p}(G)$ is S-semipermutable in $G$ for all subgroups $H \unlhd P$ with $|H|=d$. Then either $G$ is p-supersoluble, or else $\left|P \cap \mathrm{O}^{p}(G)\right|>d$.

We present some applications of Theorem 2. They concern the structure of a group $G$ in which the subgroups $H \cap \mathrm{O}^{p}(G)$ are S-semipermutable in $G$ for 
all subgroups $H$ of a fixed order $d$ of a given Sylow $p$-subgroup of $G$, and can be regarded as S-semipermutable versions of [2, Corollary C and Corollary E]. They can be also considered as an improvement of the following result.

Theorem 3 ([7, Theorem 3.3]). Let $P \in \operatorname{Syl}_{p}(G)$ and let $d$ be a power of $p$ such that $p \leq d<|P|$. Assume that every subgroup of $P$ with order $d$ and all cyclic subgroups of $P$ of order 4 (if $d=2$ and $P$ is not abelian) are $S$-semipermutable in $G$. Then $G$ is p-supersoluble.

We consider first the case when $d$ is a prime.

Theorem 4. Let $P \in \operatorname{Syl}_{p}(G)$. Suppose that $H \cap \mathrm{O}^{p}(G)$ is $S$-semipermutable in $G$ for all subgroups $H \leq P$ with $H$ cyclic of order $p$ or 4 (if $p=d=2$ and $P$ is not abelian). Then $G$ is p-supersoluble.

Our last result concerns the case when $p<d<|P|$.

Theorem 5. Let $P \in \operatorname{Syl}_{p}(G)$ and let $d$ be a power of $p$ such that $p<d<|P|$. Suppose that $H \cap \mathrm{O}^{p}(G)$ is S-semipermutable in $G$ for all subgroups $H \leq P$ with $|H|=d$. Then $G$ is p-supersoluble.

We mention that Corollary $\mathrm{C}$ and Corollary E of [2] by Guo and Isaacs are immediate consequences of Theorems 4 and 5. Note that the proof of Corollary E in [2] is incomplete.

\section{Proofs}

Proof of Theorem 2. Assume the result is not true and let $G$ be a counterexample of least order. Write $U=\mathrm{O}^{p}(G), N=P \cap U$. Then $|N| \leq d$ and $G$ is not $p$-supersoluble. In particular, $N \neq 1$ and $d \geq p$. Write $\mathcal{H}=\{H \unlhd P|| H \mid=d\}$. By hypothesis, $H \cap U$ is S-semipermutable in $G$ for each $H \in \mathcal{H}$.

Step 1. $\mathrm{O}_{p^{\prime}}(G)=1$.

Write $V=\mathrm{O}_{p^{\prime}}(G)$ and assume that $V \neq 1$. Consider the factor group $\bar{G}=G / V$. Let $\bar{H}$ be a normal subgroup of $\bar{P}$ of order $d$. Then there is $H \in \mathcal{H}$ such that $\bar{H}=H V / V$. Since $H \cap U$ is S-semipermutable in $G$, we have $\bar{H} \cap \bar{U}=(H \cap U) V / V$ is S-semipermutable in $G$. Since $|\bar{U} \cap \bar{P}| \leq d$, it follows that $\bar{G}$ is $p$-supersoluble by the minimal choice of $G$. Hence $G$ is $p$-supersoluble and this is contradiction. Thus $V=1$, as required.

Step 2. $N$ is S-semipermutable in $G$.

Since $N$ is normal in $P$ of order at most $d$, there is $H \in \mathcal{H}$ such that $N \leq H \leq P$. Then $N=H \cap U$ is S-semipermutable in $G$. 
Step 3. Let $Y$ be a maximal subgroup of $P$. Then $Y \cap U=Y \cap N$ is S-semipermutable in $G$.

Since $|N| \leq d$, there is an $H \in \mathcal{H}$ such that $Y \cap N \leq Y \cap U \leq H \leq Y$. Then $Y \cap N=Y \cap N \cap H=H \cap N=H \cap P \cap U=H \cap U$. Thus $Y \cap N=Y \cap U=H \cap U$ is S-semipermutable in $G$.

Step 4. Let $\left\langle N^{G}\right\rangle$ be the normal closure of $N$ in $G$. Then $G /\left\langle N^{G}\right\rangle$ is p-nilpotent. In particular, $G$ is p-soluble.

Since $\left\langle N^{G}\right\rangle \leq U$ and $\left|U:\left\langle N^{G}\right\rangle\right|$ divides the $p^{\prime}$-number $|U: N|$, we have that $U /\left\langle N^{G}\right\rangle$ is a $p^{\prime}$-group. Moreover, $G / U$ is a $p$-group. It follows that $G /\left\langle N^{G}\right\rangle$ is $p$-nilpotent. On the other hand, we know that $G=P U$ and, by Step 2, $N$ permutes with each Sylow $q$-subgroup of $U$ for every prime $q \neq p$. By [4, Theorem A], the normal closure $\left\langle N^{G}\right\rangle$ of $N$ in $G$ is soluble. We conclude that $G$ is $p$-soluble.

Step 5. A final contradiction.

Assume that $d=p$. Then $N$ is a normal Sylow $p$-subgroup of $U$ by Steps 1 and 4 . Hence $G$ is $p$-supersoluble, against the choice of $G$. Thus $d>p$. Let $T$ be a minimal normal subgroup of $G$ contained in $U$. By Steps 1 and 4 , $T \leq N$. Thus $|T| \leq d$. Suppose that $|T|<d$. We argue that $G / T$ satisfies the hypotheses of the theorem. Clearly, $1 \leq d /|T|<|P / T|$. Let $H / T$ be a normal subgroup of $P / T$ of order $d /|T|$. Then $H \in \mathcal{H}$. It follows that $H / T \cap \mathrm{O}^{p}(G / T)=H / T \cap U / T=(H \cap U) / T$, which is S-semipermutable in $G / T$. This shows that $G / T$ satisfies the hypotheses of the theorem, as claimed. Since $|P / T \cap U / T|=|(P \cap U) / T| \leq d /|T|$, it follows that $G / T$ is $p$-supersoluble by minimality of $G$. By [3, Kapitel VI, Satz 8.6], we may suppose that $T \not \subset \Phi(P)$. Let $Y$ be a maximal subgroup of $P$ such that $T \not \leq Y$. By Step 3, $Y \cap N$ is S-semipermutable in $G$, and so $(Y \cap N) Q$ is a subgroup of $G$ for every Sylow $q$-subgroup $Q$ of $G$ with $q \neq p$. Since $T$ is a normal $p$-subgroup of $G$, it follows that $T \cap(Y \cap N) Q=T \cap Y \cap N=T \cap Y$, which is normal in $(Y \cap N) Q$. Then $T \cap Y$ is normalised by $Q$. It follows that $T \cap Y$ is normalised by $U$. Since $T \cap Y$ is a normal subgroup of $P$, we have that $T \cap Y \unlhd G$. This implies that $T \cap Y=1,|T|=p$, and so $G$ is $p$-supersoluble, which is a contradiction.

Let $|T|=d$. Then $T=N$. By Step $4, G / T$ is $p$-nilpotent. Since the class of all $p$-nilpotent groups is a saturated formation by [3, Kapitel VI, Beispiele 7.6], $T$ is a proper subgroup of $P$ which is not contained in $\Phi(G)$. Let $Y$ be a maximal subgroup of $P$ such that $T \not \leq Y$. By Step 3, $N \cap Y=$ $T \cap Y$ is S-semipermutable in $G$. Arguing as in the previous paragraph, we obtain that $Y \cap T=Y \cap N$ is normal in $G$. This implies that $T \cap Y=1$ and $|T|=p$, against our assumption $|T|=d>p$. This final contradiction completes the proof. 
Proof of Theorem 4. We proceed by induction on $|G|$. Write $U=\mathrm{O}^{p}(G)$, $U_{p}=P \cap U$. We may suppose that $\mathrm{O}_{p^{\prime}}(G)=1$. By Theorem 2, we may suppose that $\left|U_{p}\right|>p$. If $G=U$, then each subgroup of order $p$ or 4 is Ssemipermutable in $G$. Applying Theorem 3, we get that $G$ is $p$-supersoluble. Therefore, we may assume that $U$ is a proper subgroup of $G$. Clearly $U=$ $\mathrm{O}^{p}(U)$ and every subgroup of order $p$ or 4 of $U_{p}$ is S-semipermutable in $U$ by [1, Lemma 1.2.7]. By the induction hypothesis, $U$ is $p$-supersoluble. Since $\mathrm{O}_{p^{\prime}}(U)=1$, it follows that $U_{p}$ is normal in $U$ by [1, Lemma 1.2.16]. Hence $U_{p}$ is a normal subgroup of $G$. Assume that $p=2$. Then $U$ is 2-nilpotent and so it is a 2-group. This implies that $G$ is a 2-group. Therefore we may suppose that $p>2$. Then every chief factor of $G$ below $U_{p}$ is cyclic, by [6, Theorem 3.3]. Since $G / U_{p}$ has a normal Hall $p^{\prime}$-subgroup, we conclude that $G$ is $p$-supersoluble.

The proof of Theorem 5 depends on the following lemmas. The first one is the S-semipermutable version of [2, Corollary C].

Lemma 6. Let $P \in \operatorname{Syl}_{p}(G)$ with $|P|>p$. Suppose that, for every maximal subgroup $H$ of $P, H \cap \mathrm{O}^{p}(G)$ is S-semipermutable in $G$. Then $G$ is p-supersoluble.

Proof. With a contradiction in mind, assume that $G$ is not $p$-supersoluble. Write $U=\mathrm{O}^{p}(G)$. By Theorem 2, $P \cap U=P$, that is, $P \leq U$, and so $G=U$. This means that every maximal subgroup of $P$ is S-semipermutable in $G$. Applying [7, Theorem 3.2], we conclude that $G$ is $p$-supersoluble.

Lemma 7. Let $P \in \operatorname{Syl}_{p}(G)$ and write $U=\mathrm{O}^{p}(G)$. Suppose that $U_{p}=P \cap U$ is a normal p-subgroup of $G$ and that $d$ is a power of $p$ such that $p<d<\left|U_{p}\right|$. Suppose also that $H$ is $S$-permutable in $G$ for all subgroups $H \leq U_{p}$ with $|H|=d$. Then $G$ is p-supersoluble.

Proof. We proceed by induction on $|G|$. We may suppose that $\mathrm{O}_{p^{\prime}}(G)=1$. If $U=G$, we can apply Theorem 3 to conclude that $G$ is $p$-supersoluble. Therefore we may assume that $U$ is a proper subgroup of $G$. Since $U=\mathrm{O}^{p}(U)$ and every subgroup of order $d$ of $U_{p}$ is S-permutable in $U$ by [1, Lemma 1.2.7], we have that $U$ is $p$-supersoluble. If $p=2$, we can argue as in Theorem 4 to conclude that $G$ is a 2 -group. Hence we may assume $p>2$.

Let $T$ be a minimal normal subgroup of $G$ contained in $U_{p}$. Assume that $|T|>d$. Let $H$ be a normal subgroup of $P$ such that $H \leq T$ and $|H|=d$. Since $H$ is S-permutable in $G$, we have that $U \leq \mathrm{N}_{G}(H)$ by [1, Lemma 1.2.16]. Therefore $G=U P \leq \mathrm{N}_{G}(H)$, that is, $H$ is a normal subgroup of $G$. Since $T$ is a minimal normal subgroup of $G$, we conclude that $H=1$, against our 
assumption $|H|=d>p$. Therefore $|T| \leq d$. We focus now on $G / T$ and prove that it is $p$-supersoluble.

Assume that $|T|<d$. Then $H / T$ is S-permutable in $G / T$ for each $H / T \leq$ $U_{p} / T$ with $|H / T|=d /|T|$. If $d /|T|>p$, then $G / T$ is $p$-supersoluble by induction. If $d /|T|=p$, the conclusion follows from Theorem 4 .

Assume now that $|T|=d$. Therefore we may assume that $p$ is odd. Bearing in mind Lemma 6 , we may suppose that $\left|U_{p}\right|>p|T|=p d$. Let $K$ be a subgroup of $U_{p}$ such that $|K / T|=p$. Since $T$ is non-cyclic, there is a maximal subgroup $L$ of $K$ such that $K=T L$. Then $L$ is of order $d$ and so it is S-permutable in $G$. By [1, Lemma 1.2.7], $K / T$ is S-permutable in $G / T$. By Theorem 4, $G / T$ is $p$-supersoluble.

Since $G / T$ is $p$-supersoluble and the class of all $p$-supersoluble groups is a saturated formation by [3, Kapitel VI, Hilfssatz 8.3], we may assume that $T$ is the unique minimal normal subgroup of $G$ contained in $U_{p}$ and $\Phi(G) \cap U_{p}=1$. By [3, Kapitel III, Satz 4.5], $U_{p}$ is a direct product of some minimal normal subgroups of $G$. Thus $\left|U_{p}\right|=|T|$, against the hypothesis $|T| \leq d<\left|U_{p}\right|$. This final contradiction completes the proof.

Proof of Theorem 5. We may suppose that $\mathrm{O}_{p^{\prime}}(G)=1$. By Theorem 2, we may suppose that $|P \cap U|>p$. If $G=U$, then each subgroup of order $d$ is S-semipermutable in $G$. By Theorem 3, the conclusion follows. Suppose that $U<G$. By [1, Lemma 1.2.7], the hypotheses of the theorem hold in $U$. By induction, $U$ is $p$-supersoluble. Since $\mathrm{O}_{p^{\prime}}(U)=1$, we can apply [1, Lemma 1.2.16] to conclude that $U_{p}=U \cap P$ is a normal subgroup of $G$. Therefore all subgroups of order $d$ are S-permutable in $G$ by [7, Lemma 2.2]. Applying Lemma 7, we conclude that $G$ is $p$-supersoluble.

\section{Acknowledgements}

The first and the second authors have been supported by the grant MTM201454707-C3-1-P from the Ministerio de Economía y Competitividad, Spain, and FEDER, European Union. The first author has been also supported by NSC of China(No. 11271085) and a project of Natural Science Foundation of Guangdong Province(No. 2015A030313791). The third author was supported by NSF of China(No. 11201082), Cultivation Program for Outstanding Young College Teachers (Yq2013061) and Project(2013B051000075) of Guangdong Province, Pei Ying Yu Cai Project of GDUT. The third author also thanks the China Scholarship Council and the Departament d'Àlgebra of the Universitat de València for its hospitality. 


\section{References}

[1] A. Ballester-Bolinches, R. Esteban-Romero, and M. Asaad. Products of finite groups, volume 53 of de Gruyter Expositions in Mathematics. Walter de Gruyter, Berlin, 2010.

[2] Y. Guo and I. M. Isaacs. Conditions on $p$-subgroups implying $p$-nilpotence or p-supersolvability. Arch. Math. (Basel), 105:215-222, 2015.

[3] B. Huppert. Endliche Gruppen I, volume 134 of Grund. Math. Wiss. Springer Verlag, Berlin, Heidelberg, New York, 1967.

[4] I. M. Isaacs. Semipermutable $\pi$-subgroups. Arch. Math. (Basel), 102:1-6, 2014 .

[5] O. H. Kegel. Sylow-Gruppen und Subnormalteiler endlicher Gruppen. Math. Z., 78:205-221, 1962.

[6] Y. Li and B. Li. On weakly s-supplemented subgroups of finite groups. J. Algebra Appl., 10:1-10, 2011.

[7] Y. Li, S. Qiao, N. Su, and Y. Wang. On weakly s-semipermutable subgroups of finite groups. J. Algebra, 371:250-261, 2012.

[8] A. N. Skiba. On weakly s-permutable subgroups of finite groups. $J$. Algebra, 315(1):192-209, 2007.

[9] L. Wang and Y. Wang. On $s$-semipermutable maximal and minimal subgroups of Sylow p-subgroups of finite groups. Comm. Algebra, 34:143$149,2006$. 